\title{
Gestational diabetes mellitus and progression to type 2 diabetes
}

\author{
Delia loana CUDALBA ${ }^{1}$, Nicolae GICA ${ }^{1,2}$, Radu BOTEZATU ${ }^{1,2}$, Corina GICA $^{1}$, Anca Marina CIOBANU $^{1}$, \\ Brindușa Ana CIMPOCA ${ }^{1}$, Alina VEDUTA ${ }^{1}$, Gheorghe PELTECU ${ }^{1,2}$, Anca Maria PANAITESCU ${ }^{1,2}$ \\ ${ }^{1}$ Filantropia Clinical Hospital, Bucharest, Romania \\ 2 "Carol Davila" University of Medicine and Pharmacy, Bucharest, Romania
}

\begin{abstract}
Diabetes mellitus is a metabolic disease with life-threatening morbidity and with epidemic spread. This metabolic disorder affects an increasing number of pregnant women. General practitioners and obstetricians need to know how some occult maternal metabolic disorders could be revealed by pregnancy and how pregnancy could influence the fetal health and the long-term progression of maternal disease. Pregnancy is considered a stress test for life and diabetes mellitus is a good example to illustrate this correlation.
\end{abstract}

Keywords: gestational diabetes mellitus, pregnancy, type 2 diabetes

\section{INTRODUCTION}

Diabetes mellitus is an emerging epidemic associated with life-threatening morbidity. According to World Health Organization (WHO), approximately 422 million people are suffering from diabetes worldwide - that is 1 person in 11 [1]. National Diabetes Statistics Report estimated for 2018 that 34.2 million people, $10.5 \%$ of the US population, had diabetes, 26.9 million being diagnosed and 7.3 million undiagnosed [2].

Diabetes complications include cardiovascular disease, peripheral artery disease, stroke, neuropathy, renal dysfunction and retinopathy and are responsible for significant morbidity and mortality, reducing life expectancy by 10 years [3]. Worldwide, diabetes kills about 3.4 million people annually [1]. These statistics underscore the magnitude of determining high risk population and improving prevention programs.

One of the high-risk groups include women who develop glucose intolerance during pregnancy. Gestational diabetes mellitus (GDM) occurs in nearly $14 \%$ of live births [4]. Even if glucose intolerance usually withdraws after birth, for women with prior GDM the risk of lifetime type 2 diabetes mellitus (T2DM) is 7-fold higher than in women with normoglycemic pregnancies. They have double risk of being diagnosed with T2DM in the first months after giving birth $[5,6]$ and up to $50 \%$ of them develop T2DM within 5 years postpartum [6].

\section{AIM}

This paper aims to review literature and current studies on the incidence of T2DM among women with gestational diabetes history, to identify risk factors associated with the onset of overt diabetes in this category and to assess what could improve this situation.

\section{METHODS}

A systematic literature electronic search for reviews and guidelines was undertaken using major electronic databases and the official websites of diabetes and obstetrics and gynecology associations. Search words 
were "gestational diabetes mellitus", 'type 2 diabetes mellitus". Publications were selected based on accessibility to full paper article, quality evaluation, publication year, trying to select recent studies. References from the chosen studies were scrutinized in order to identify other relevant articles. The publications used are mentioned in References section. The guidelines and statistics papers selected were the last one published.

\section{RESULTS}

Beyond recommendations regarding the management of GDM, current guidelines propose specific post-partum measures. In the last edition of its guideline American Diabetes Association (ADA) recommends screening women with the 75-g oral glucose tolerance test at 4-12 weeks postpartum, using non pregnancy diagnostic criteria (diabetes is diagnosed at 2 hours blood sugar greater than or equal to $200 \mathrm{mg} / \mathrm{dl}$, prediabetes for values between $140 \mathrm{mg} / \mathrm{dl}$ and $199 \mathrm{mg} / \mathrm{dl}$ ) [7].

Therefore, the first assessment should be done at 4-12 weeks postpartum. From the screening tools, the preferred one is the 75-g oral glucose tolerance test, which is considered superior over A1C. If glycemic values are normal, lifelong screening is recommended every 1-3 years: annual fasting plasma glucose or A1C or triennial 75-g OGTT [7].

American Diabetes Association mentions the risk of the onset of T2DM over a history of GDM. Factors lowering the risk of progression are considered adopting healthier diets, intensive lifestyle, pharmacological treatment. These adjustments proved to reduce progression to diabetes by $35-40 \%$ over 10 years [8].

Regarding screening method, postpartum check-up and lifelong surveillance, ACOG recommendations are similar to the ones published by ADA [9].

The first meta-analysis to assess the incidence of T2DM in women with prior GDM was published in the Journal of Diabetes Research in April 2020. Twenty-eight studies including 170,139 women with history of GDM were selected and 34,627 cases of T2DM were identified. The incidence of T2DM after GDM was 26.20 per 1,000 person-years. Geographical patterns of the incidence showed significant differences. The highest incidence rate was from Asian women - 45.96 per 1,000 person-year. North America had the next higher rate (25.22 per 1000 person-year), followed by Europe (25.17 per 1,000 person-year) and Australasia (18.12 per 1,000 person-years). Risk factors for the onset of T2DM were older age and higher body mass. Regarding the follow-up of the patients, this study showed that the risk increased with $9.6 \%$ o per year $-19.72 \%$ at 10 years, $29.36 \%$ at 20 years, $39.00 \%$ at 30 years, $48.64 \%$ at 40 years, and $58.27 \%$ at 50 years [10].
A systematic review and meta-analysis of 39 studies including 95,750 women assessed the risk of progression to T2DM for women with GDM, taking into account individualized risk estimates. Among maternal characteristics, higher BMI (a high BMI doubled the risk of future T2DM), advanced maternal age, family history of diabetes, non-white ethnicity, were associated with higher risk of T2DM. Pregnancy-specific factors which increased the risk, were early diagnosis of GDM, use of insulin in order to manage GDM, high level of fasting glucose and increased $\mathrm{HbA} 1 \mathrm{c}$. Preterm delivery, hypertensive disorders in pregnancy and multiparity were associated with higher risk to develop T2DM in the future. Macrosomia, gestational weight gain and breastfeeding did not increase the risk [11].

Beyond the "seven-fold" risk increase in developing diabetes after GDM are clinical and genetic risk factors, which attribute to each case a different progression over time. A few studies tried to find and evaluate the impact of these parameters to the onset and evolution of this disease.

Regarding the onset of type 2 diabetes mellitus patients could be divided in early converters, which evolve to T2DM in a few months postpartum, and late converters, when glucose metabolism is altered more than one year after giving birth. For the first category of converters the clinical factors associated with a higher tendency to diabetes were altered beta-cell function, lower fasting insulin concentration, higher levels of glucose at the antepartum OGTT and higher body mass index before the pregnancy. From those, only the last two ones were found to affect the late converters. Both types had obesity as risk factor [12].

Concerning the genetic profile for diabetes, the genotypes found to be involved in the progression of GDM to diabetes were the HHEX gene (hematopoetically expressed homeobox), CDKN2A and CDKN2B genes and CDKAL1gene. The HHEX gene is responsible for a transcription factor involved in pancreatic development $(32,33), C D K N 2 A$ and $C D K N 2 B$ genes are involved in beta-cell replication $(34,35)$ and CDKAL1 gene associate with variants of diabetes with altered insulin secretion. Late converters had variants in CDKAL1 and early converters associated the other ones mentioned [12].

It is documented that metabolic syndrome is an independent risk factor for T2DM in women with prior GDM [13].

Regarding predisposing conditions for developing GDM during pregnancy, studies show that women diagnosed with polycystic ovary syndrome (PCOS) have higher risk for the onset of GDM. Usually, women with PCOS have up to 8-fold higher risk of T2DM. A study examining 988 cases of women with PCOS who developed GDM assessed the risk of T2DM. There was a risk 
of diabetes of $7.1 \%$ for women with PCOS and GDM which did not require pharmacological treatment and almost fourfold higher risk (27\%) in the cases that required medication [14].

A study published in 2019 analyzed 12,622 women with GDM between 2006-2012 focusing on 1091 women with GDM who had a second delivery within 3 years of their index delivery and aimed to assess the impact of time interval between deliveries on recurrence of GDM and early T2DM onset. In $49.3 \%$ of cases, GDM recurred regardless of the interval to the next pregnancy. Recurrence tripled the odds of early T2DM onset within 3 years of the second delivery [15].

\section{DISCUSSION}

Determining postpartum modifiable risk factors, continuous engagement of the patients in education and increasing patient awareness in healthcare practice, plays a major role in the prevention of type 2 diabetes mellitus among women with prior gestational diabetes.

Women with prior GDM should be considered high risk patients for developing T2DM. Guidelines recommend screening - postpartum and lifelong afterwards, early identification, referral to primary care, lifestyle interventions, such as physical activity and healthful dietary patterns and anti-glycemic agents.

Optimizing health behaviors, healthful dietary change and regular physical activity, proved to significantly modify the risk of type 2 diabetes.

One study, which followed 4,413 women with prior GDM, used 3 dietary pattern adherence scores usually used in general population (The alternate Mediterranean Diet, Dietary Approaches to Stop Hypertension, and alternate Healthy Eating Index) and assessed the impact of life-long dietary modifications. All of the patterns associated lower risk of T2DM, between 40 and $57 \%[16]$.

The Diabetes Prevention Program conducted a randomized, controlled clinical trial comprising 2190 patients, 350 providing a past history of GDM and 1416 with no prior history of GDM, with the purpose of evaluating the effects of lifestyle intervention and pharmacological treatment. Both categories started from a similar glucose level. Women with history of GDM which were not randomized to medication or lifestyle intervention, had an incidence rate of diabetes $71 \%$ higher than women with no history of GDM. Among women with prior GDM, both intensive lifestyle and metformin therapy reduced the incidence of diabetes by $50 \%$ [17].

Among adopting a healthy lifestyle and pharmacological treatment, another aspect recommended by only few guidelines, because of the lack of available ev- idence, is lactation. Studies showed that in women with previous GDM breastfeeding more than 4-12 weeks postpartum lower their risk for T2DM. The etiology of the beneficial effect of lactation is poorly understood. There are some hypotheses which count on the effect of prolactin on pancreatic beta-cell formation and function, visceral fat mobilization or the role of milk production in spending extra energy [18].

The diagnosis of GDM determines a particular management and a constant pregnancy follow-up associated with different interventions in order to lower maternal fetal risks. Although being aware of the risks generated by GDM history, concern resumes only to measures taken during the pregnancy for the wellbeing of the off-spring as well as the mother's, with low post-partum follow-up rates.

In the UK there are reported rates of long-term follow-up in primary care around 20\% [19].

A study followed 12,622 women with GDM 3 years post-delivery and identified low rates of glucose testing and transition to primary care: $5.8 \%$ of women went for postpartum screening, $21.8 \%$ monitored their glucose level at 1 year and $51 \%$ at 3 years. Concerning the transfer to primary care, $5.7 \%$ had contact with primary care at 6 months, $13.2 \%$ at 1 year and $40.5 \%$ at 3 years [20].

The barriers in lowering the risk of T2DM after GDM could be gaps in the health system - the discontinuation between obstetric services and primary care and practical, social, environmental and financial factors. Pregnancy motivates health behavior change because of the concern for the future baby's health, but post-partum these modifications are not being maintained. The impediments to a healthy postnatal lifestyle were considered to be the lack of time, as healthy eating - preparing healthy meals and exercise are considered time-consuming activities, women preferring to prioritize their newborn.

\section{CONCLUSIONS}

The progression to type 2 diabetes among women with prior gestational diabetes is determined by multiple factors and is significantly altered by lifestyle factors. The high incidence of T2DM after GDM provides evidence for the importance of screening and prevention and should motivate both health care providers and women toward taking action.

The occasion of constant medical contact given by the pregnancy should be seen as an opportunity to influence the health of both mother and off-spring.

Measures that could improve the life-time follow-up of women with history of GDM involves firstly establishing a data linkage and communication between secondary and primary care providers. After the 
delivery there should be a responsibility transfer between obstetrician and primary care physician and an attempt to fill this lack of communication.

Studies suggested that providing clear information before birth regarding the dietary changes postnatally, was beneficial for women. Post-delivery counselling should include personalized risk communication. Women tend to be more motivated to take informed choices regarding diet and lifestyle changes if they understand their individual risk of future onset of diabetes.

Considering the low rates follow-up and prophylactic measures, there is need to improve patient's awareness on gestational diabetes, type 2 diabetes, their Conflict of interest: none declared Financial support: none declared risks and complications and to encourage attitude and prevention.

This review is an invitation for health care providers to reconsider the follow-up of this high-risk group. The patients' attitude toward this matter and with that the natural course of a disease can only be changed by public health efforts and physicians.

\section{Acknowledgement}

All authors equally contributed to this article.

\section{REFERENCES}

1. World Health Organization. https://www.who. int/news-room/fact-sheets/detail/diabetes.

2. National Diabetes Statistics Report, 2020.

3. Department of Health. National service framework for diabetes. London (2001). https://www.gov.uk/government/publications/ national-serviceframework-diabetes.

4. International Diabetes Federation. IDF diabetes atlas, 7th edition. http://www. diabetesatlas.org/.

5. Casas JP, Hingorani AD, Williams D. Bellamy L, etal. Type 2 diabetes mellitus. Lancet. 2009;373:1773-1779.

6. Newton KM, Knopp RH. Kim C, et al. Gestational diabetes and the incidence of type 2 diabetes: a systematic review. Diabetes Care. 2002;25:1862-1868.

7. American Diabetes Association. Management of Diabetes in Pregnancy: Standars of Medical Care in Diabetes 2020. Diabetes Care. 2020;43(Suppl. 1): S183-S192.

8. Christophi CA, Edelstein SL, Aroda VR, et al. Diabetes Prevention Program Research Group.The effect of lifestyle intervention and metformin on preventing or delaying diabetes among women with and without gestational diabetes: the Diabetes Prevention Program outcomes study 10-year follow-up. Clin Endocrinol Metab. 2015;100:1646-1653.

9. The American College of Obstetricians ang Gynecologists. Gestational Diabetes
Mellitus. ACOG Practice Bulletin 2018;131(2):e57-e58.

10. Li Z, Cheng Y, Wang D, Chen H, Chen H, Ming WK, Wang Z. Incidence Rate of Type 2 Diabetes Mellitus after Gestational Diabetes Mellitus: A Systematic Review and Meta-Analysis of 170,139 Women. J Diabetes Res. 2020 Apr 27;2020:3076463.

11. Hashi AA, Zamora J, Rayanagoudar G, et al. Quantification of the type 2 diabetes risk in women with gestational diabetes: a systematic review and meta-analysis of 95,750 women. Diabetologia 2016; 59:1403-1411.

12. Sung Hee Choi, Hye Seung Jung, Soo Heon Kwak, et al. Clinical and Genetic Risk Factors for Type 2 Diabetes at Early or Late Post Partum After Gestational Diabetes Mellitus. J Clin Endocrinol Metab. 2013;98(4):E744-E752.

13. Chang Ho Ahn, Joon Ho Moon, Nam H. Cho, et al. Metabolic syndrome independently predicts future diabetes in women with a history of gestational diabetes mellitus. Medicine. 2016;95:35:e4582.

14. Lo JC, Yang J, Gunderson EP, Hararah MK, Gonzalez JR, Ferrara A. Risk of Type 2 Diabetes Mellitus following Gestational Diabetes Pregnancy in Women with Polycystic Ovary Syndrome. J Diabetes Res. 2017;2017:5250162.

15. Bernstein J, Lee-Parritz A, Quinn E, Ameli O, Craig $M$, Heeren $T$, Iverson $R$, Jack $B$,
McCloskey L. After Gestational Diabetes: Impact of Pregnancy Interval on Recurrence and Type 2 Diabetes. Biores Open Access. 2019;8(1):59-64.

16. Hu FB, Chavarro J, Tobias DK, et al. Healthful dietary patterns and type 2 diabetes risk among women with a history of gestational diabetes. Arch Intern Med. 2012;172(20):1566-1572.

17. Christophi CA, Metzger BE, Ratner RE, et al. Prevention of Diabetes in Women with a History of Gestational Diabetes: Effects of Metformin and Lifestyle Interventions. J Clin Endocrinol Metab. 2008;93:4774-4779,.

18. Arata N, Kawasaki M, Tanase-Nakao K, et al. Potential protective effect of lactation against incidence of type 2 diabetes mellitus in women with previous gestational diabetes mellitus: A systematic review and metaanalysis. Diabetes Metab Res Rev. 2017;33:e2875.

19. McGovern A, Butler L, Jones S, van Vlymen J, Sadek K, Munro N, Carr H, de Lusignan $\mathrm{S}$. Diabetes screening after gestational diabetes in England: a quantitative retrospective cohort study. $\mathrm{Br} J$ Gen Pract. 2014;64(618):e17-23.

20. Quinn E, Ameli O, Bernstein JA, et al. Follow-up after gestational diabetes: a fixable gap in women's preventive healthcare. BMJ Open Diab Res Care. 2017;5:e000445. 